INSTITUTE OF FOOD AND RESOURCE ECONOMICS

MSAP Working Paper Series

No. $01 / 2011$

A Unifying Framework for the Problem of Adjudicating Conflicting

Claims.

Jens Leth Hougaard

Institute of Food and Resource Economics

University of Copenhagen

Juan D. Moreno-Ternero

Department of Economics

Universidad de Malaga and Pablo de Olavide

Lars Peter Østerdal

Department of Economics

University of Copenhagen

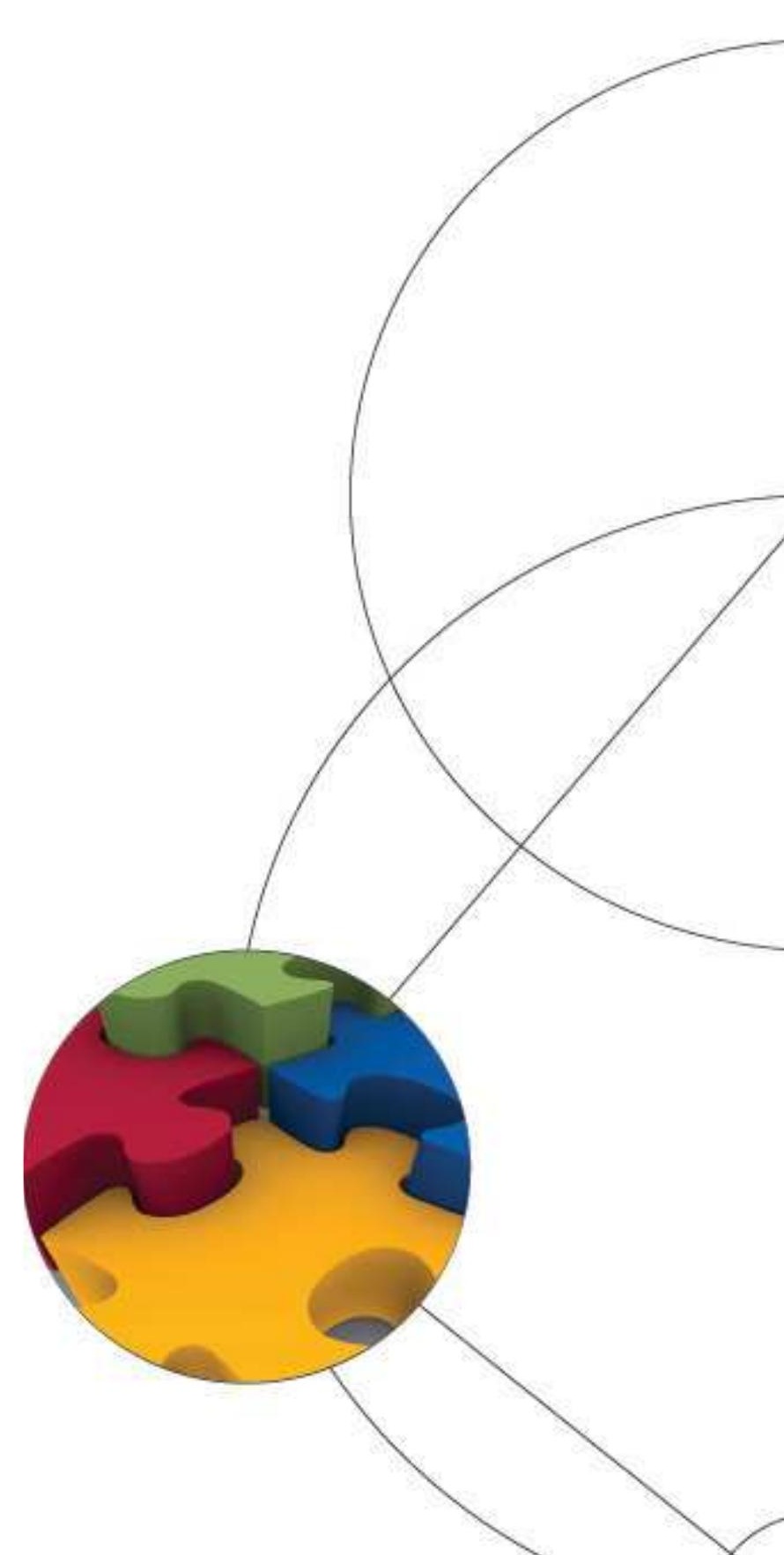




\title{
A unifying framework for the problem of adjudicating
}

$$
\text { conflicting claims* }
$$

\section{Jens L. Hougaard ${ }^{\dagger} \quad$ Juan D. Moreno-Ternero ${ }^{\ddagger} \quad$ Lars P. Østerdal $^{\S}$}

January 30, 2011

\begin{abstract}
In a recent paper, Thomson and Yeh [Operators for the adjudication of conflicting claims, Journal of Economic Theory 143 (2008) 177-198] introduced the concept of operators on the space of rules for the problem of adjudicating conflicting claims. They focussed on three operators in order to uncover the structure of such a space. In this paper, we generalize their analysis upon presenting and studying a general family of operators inspired by three apparently unrelated approaches to the problem of adjudicating conflicting claims. We study the structural properties of this family and show, in particular, that most of Thomson and Yeh's results are specific cases of our study.
\end{abstract}

JEL numbers: D63.

Keywords: rationing, operators, baselines, composition, claims.

${ }^{*}$ Financial support from the Spanish Ministry of Science and Innovation (ECO2008-03883) and from Junta de Andalucía (P08-SEJ-04154) is gratefully acknowledged.

${ }^{\dagger}$ University of Copenhagen.

¥Universidad de Málaga, Universidad Pablo de Olavide, and CORE, Université catholique de Louvain.

$\S$ University of Copenhagen. 


\section{Introduction}

The problem of adjudicating conflicting claims describes a situation in which an arbitrator has to allocate a given amount of a perfectly divisible (and homogeneous) resource among a group of agents when the available amount is not enough to fully honor their claims. Most rationing problems can be given this form (e.g., the division of an estate that is insufficient to cover all the debts incurred by the deceased, the collection of a given tax from taxpayers, sharing the cost of a public facility). The reader is referred to Moulin (2002) or Thomson (2003) for reviews of the sizable related literature initiated by O'Neill (1982). In most of this literature the aim is to single out rules that assign for each problem an allocation indicating how much each agent obtains. A recent study by Thomson and Yeh (2008) uncovers the structure of the space of rules upon studying operators on such a space. An operator is a mapping on the space of rules that associates with each rule another one. Thomson and Yeh (2008) consider three operators. First, the duality operator, which assigns to each rule $R$, its dual $\left(R^{d}\right)$, that allocates awards in the same way as $R$ allocates losses. Second, the so-called claims truncation operator, which assigns to each rule $R$ the rule $R^{t}$ defined, for each problem, by applying $R$ after each claim has been truncated at the available amount. Third, the attribution of minimal rights operator, which associates with each rule $R$ the rule $R^{m}$ defined by the following two-step procedure. For each problem, first each agent receives her minimal right (to be understood as the part of the available amount that remains, if anything, when all other agents have been fully honored); second, each agent gets an award according to $R$ applied to the revised problem obtained by reducing agents' claims by their minimal burdens, and the available amount by the sum of the minimal rights. Thomson and Yeh (2008) establish a number of results linking them and determine which properties of rules are preserved under each of these operators, and which are not.

In this paper, we generalize the analysis of Thomson and Yeh (2008) upon providing a systematic analysis of a family of operators generalizing the last two mentioned above. Our family is inspired by three apparently unrelated approaches to the problem of adjudicating conflicting claims that, as we shall see later, are intimately connected. First, the so-called baseline rationing, a generalization of the benchmark model upon adding a vector in the awards space representing some reference point, or baseline, judged relevant to the division (see Hougaard et al., (2010) for further details). Second, two of the principles most frequently employed in the literature on the problem of adjudicating conflicting claims, pertaining to the way rules react 
to tentative allocations of the available amount, and known as composition properties (e.g., Young, 1988; Moulin, 2000). Third, the analysis of lower bounds, an important aspect within the theory of fair allocation, (e.g., Thomson, 2007) that has only been recently explored for the problem of adjudicating conflicting claims (e.g., Moreno-Ternero and Villar, 2004; Dominguez and Thomson, 2006).

More precisely, think of the following situation: after having divided the allocation of the available amount among its creditors, it turns out that the actual value of the amount is larger than was initially assumed. Then, two options are open: either the tentative division is canceled altogether and the actual problem is solved, or we add to the initial distribution the result of applying the rule to the remaining amount. The requirement of composition up is that both ways of proceeding should result in the same awards vectors. Think now of the dual case. Namely, after having divided the available amount among its creditors one finds that the actual value of the amount to divide falls short of what was assumed. Here again we can ignore the initial division and apply the rule to the revised problem, or we can apply the rule to the problem in which the initial claims are substituted by the (unfeasible) allocation initially proposed. The requirement of composition down is that both ways of proceeding should result in the same awards vectors.

A simple examination of the two composition principles described above will suffice to detect a close relationship between the attribution of minimal rights operator and the principle of composition up, and the claims truncation operator and the principle of composition down, respectively. In the former case, one just needs to interpret minimal rights as a baseline reflecting the tentative allocation of the available amount, whereas in the latter case, truncated claims would refer to a baseline reflecting the unfeasible allocation initially proposed. We propose in this paper a family of operators to generalize those two upon associating to each baseline an operator reflecting the composition principles with respect to it.

In other words, all operators within our family will share a common feature inspired by the composition properties. Namely, if all (individual) baselines cannot be granted, then no agent will be awarded above her baseline. Similarly, if all baselines could be granted, then each agent will be at least awarded with her baseline. More precisely, each rule will be mapped into a new rule that will solve problems according to a two-stage process, depending on whether the available amount in each problem is above or falls short the aggregate baseline for that problem. In the former case, and inspired by the axiom of composition up, the resulting rule assigns first to each agent her baseline and then adds to that distribution the result of applying 
the original rule to the remaining amount, once claims have been adjusted. In the latter case, and inspired by the axiom of composition down, the resulting rule solves each problem upon applying the original rule to the problem in which the initial claims are substituted by the (unfeasible) allocation proposed by the baselines profile.

As one can easily infer, both the claims truncation operator, and the attribution of minimal rights operator are specific members of the family of (baseline) composition operators described above. We shall study some logical relations among the operators within this family, as well as a number of results linking them, and determine which properties of rules are preserved under each of these operators, and which are not. In doing so, we shall generalize and scrutinize the results by Thomson and Yeh (2008). We shall also provide a thorough study of some other focal members of the family.

The rest of the paper is organized as follows. In Section 2, we describe the model and basic definitions. In Section 3, we introduce the family of composition operators. In Section 4, we relate the operators by means of several relationships among them. In Section 5, we explore the preservation of axioms under these operators.

\section{Model and basic concepts}

\subsection{The benchmark framework}

We study claims problems in a variable population model. The set of potential claimants, or agents, is identified with the set of natural numbers $\mathbb{N}$. Let $\mathcal{N}$ be the set of finite subsets of $\mathbb{N}$, with generic element $N$. We denote by $\mathbb{R}_{+}^{N}$ the cross-product of $|N|$ copies of $\mathbb{R}_{+}$indexed by the members of $N .^{1}$ For each $i \in N$, let $c_{i} \in \mathbb{R}_{+}$be $i$ 's claim and $c \equiv\left(c_{i}\right)_{i \in N}$ the claims profile. $^{2}$ A problem is a triple consisting of a population $N \in \mathcal{N}$, a claims profile $c \in \mathbb{R}_{+}^{N}$, and an amount to be divided $E \in \mathbb{R}_{+}$such that $\sum_{i \in N} c_{i} \geq E$. Let $C \equiv \sum_{i \in N} c_{i}$. To avoid unnecessary complication, we assume $C>0$. Let $\mathcal{D}^{N}$ be the set of problems with population $N$ and $\mathcal{D} \equiv \bigcup_{N \in \mathcal{N}} \mathcal{D}^{N}$

\footnotetext{
${ }^{1}$ Alternatively, the superscript $N$ may refer to a set pertaining to the agents in $N$. Whichever interpretation is intended should be unambiguous from the context.

${ }^{2}$ For each $N \in \mathcal{N}$, each $M \subseteq N$, and each $z \in \mathbb{R}^{N}$, let $z_{M} \equiv\left(z_{i}\right)_{i \in M}$.
} 


\subsection{Rules}

Given a problem $(N, c, E) \in \mathcal{D}$, an allocation is a vector $x \in \mathbb{R}^{N}$ satisfying that for each $i \in N$, $0 \leq x_{i} \leq c_{i}$ (boundedness) and $\sum_{i \in N} x_{i}=E$ (balancedness). A rule on $\mathcal{D}, R: \mathcal{D} \rightarrow \bigcup_{N \in \mathcal{N}} \mathbb{R}^{N}$, associates with each problem $(N, c, E) \in \mathcal{D}$ an allocation $R(N, c, E)$ for the problem. Some classical rules are the constrained equal-awards rule, $A$, which selects the allocation that makes awards as equal as possible, subject to the condition that no agent gets more than her claim, and the constrained equal-losses rule, $L$, which selects the allocation that makes losses as equal as possible, subject to the condition that no agent gets a negative amount. Formally, $A(N, c, E)=\left(\min \left\{c_{i}, \lambda\right\}\right)_{i \in N}$, and $L(N, c, E)=\left(\max \left\{0, c_{i}-\lambda\right\}\right)_{i \in N}$, where $\lambda>0$ is chosen, in each case, so that the balancedness condition is guaranteed. Two other prominent rules are the proportional rule, $P$, which awards agents proportionally to claims, and the Talmud rule (e.g., Aumann and Maschler, 1985), which is a compromise between the constrained equalawards and the constrained equal-losses rules. Formally, $T(N, c, E)=\left(\min \left\{\frac{1}{2} c_{i}, \lambda\right\}\right)_{i \in N}$ if $E \leq \frac{1}{2} C$ and $\left(\max \left\{\frac{1}{2} c_{i}, c_{i}-\mu\right\}\right)_{i \in N}$ if $E \geq \frac{1}{2} C$, where $\lambda$ and $\mu$ are chosen so that the balancedness condition is guaranteed.

\subsection{Operators}

An operator is a mapping on the space of rules that associates with each rule another one. Three operators have been proposed in the literature (e.g., Thomson and Yeh, 2008). The first one, amounts to the concept of duality. For any given rule $R$, the dual rule of $R$, denoted as $R^{d}$, associates with each $(N, c, E) \in \mathcal{D}, R^{d}(N, c, E) \equiv c-R(N, c, C-E)$. A rule is self-dual if it coincides with its dual. It is straightforward to see that the constrained equal awards and the constrained equal losses are dual rules and that the proportional and the Talmud are self-dual rules, i.e., $A^{d}=L, P^{d}=P, T^{d}=T$. The duality operator $O^{d}$ is the operator assigning to each rule $R$ its dual rule, i.e., $O^{d}(R)=R^{d}$. Note that the duality operator is an involution, i.e., $O^{d}\left(O^{d}(R)\right)=R$.

The second one refers to the concept of truncated claims. In some claims problems, it makes sense to postulate that the part of a claim that is above the amount to divide should be ignored. For a given problem $(N, c, E)$, we denote by $t(N, E, c)$ its corresponding vector of truncated claims, i.e., $t(N, E, c)=\left(t_{i}(N, c, E)\right)_{i \in N}$, where $t_{i}(N, c, E)=\min \left\{E, c_{i}\right\}$ for all $i \in N$. The claims truncation operator $O^{t}$ is the operator assigning to each rule $R$ the rule arising from applying $R$ to each problem once claims have been truncated. Formally, $O^{t}(R)=R^{t}$, where 
$R^{t}(N, c, E)=R(N, t(N, E, c), E)$, for all problems $(N, c, E)$.

The third one refers to the concept of minimal rights. Somewhat polar to the previous idea is the principle that a minimal amount should be ensured for each agent. The most natural amount, for each agent $i$, would be $m_{i}(N, c, E)=\max \left\{0, E-\sum_{j \in N \backslash\{i\}} c_{j}\right\}$, which is the portion of the amount to divide that is left to her when the claims of all other agents are fully honored, provided this amount is nonnegative. We interpret this amount as the minimal right of agent $i$. Let $m(N, c, E)=\left(m_{i}(N, c, E)\right)_{i \in N}$ and $M(N, c, E)=\sum_{i \in N} m_{i}(N, c, E)$. The attribution of minimal rights operator $O^{m}$ is the operator assigning to each rule $R$ the rule arising from allocating minimal rights first and applying then $R$ to the resulting problem once claims (and the available amount) have been adjusted. Formally, $O^{m}(R)=R^{m}$, where $R^{m}(N, c, E)=$ $m(N, c, E)+R(N, c-m(N, E, c), E-M(N, c, E))$, for all problems $(N, c, E)$.

\subsection{Baselines}

A baseline is a mapping associating to each problem a vector satisfying the boundedness condition, but not necessarily the balancedness condition. Formally, $b: \mathcal{D} \rightarrow \bigcup_{N \in \mathcal{N}} \mathbb{R}^{N}$, associates with each problem $(N, c, E) \in \mathcal{D}$ a vector $b(N, c, E)$ such that $0 \leq b_{i}(N, c, E) \leq c_{i}$ for all $i \in N .^{3}$ We single out two important types of baselines. A lower bound on $\mathcal{D}$, $\underline{b}: \mathcal{D} \rightarrow \bigcup_{N \in \mathcal{N}} \mathbb{R}^{N}$, is a baseline that associates with each problem $(N, c, E) \in \mathcal{D}$ a feasible vector. Formally, $\sum_{i} \underline{b}_{i}(N, c, E) \leq E$, for all $(N, c, E) \in \mathcal{D}$. On the other hand, an upper bound on $\mathcal{D}, \bar{b}: \mathcal{D} \rightarrow \bigcup_{N \in \mathcal{N}} \mathbb{R}^{N}$, is a baseline that associates with each problem $(N, c, E) \in \mathcal{D}$ an unfeasible vector. Formally, $\sum_{i} \bar{b}_{i}(N, c, E) \geq E$, for all $(N, c, E) \in \mathcal{D}$. An instance of a lower bound is the minimal rights application described above, whereas an instance of an upper bound is the truncated claims application also described above. Another interesting instance of lower bound is the one that assigns to each agent one $n$-th of her truncated claim (e.g., Moreno-Ternero and Villar, 2004). Formally, $\mu(N, c, E)=\left(\mu_{i}(N, c, E)\right)_{i \in N}$, where $\mu_{i}(N, c, E)=\frac{1}{n} t_{i}(N, c, E)=\frac{1}{n} \min \left\{c_{i}, E\right\} .{ }^{4}$ The corresponding upper bound is obtained when requiring that if agent $i$ 's claim is at most as large as the deficit $C-E$, she should receive at most $c_{i}-\frac{1}{n} c_{i}$, and otherwise, she should receive at most $c_{i}-\frac{1}{n}(C-E)$. Formally, $\hat{\mu}(N, c, E)=\left(\hat{\mu}_{i}(N, c, E)\right)_{i \in N}$, where $\hat{\mu}_{i}(N, c, E)=c_{i}-\frac{1}{n} t_{i}(N, c, C-E)=c_{i}-\frac{1}{n} \min \left\{c_{i}, C-E\right\}$.

\footnotetext{
${ }^{3}$ See Hougaard et al., (2010) for an alternative modeling of baseline rationing in which baselines are considered as arbitrary and exogenously given.

${ }^{4}$ Note that using claims instead of truncated claims while defining the bound is not a meaningful option, as the bounds so obtained would not necessarily be feasible for all problems.
} 
Other lower bounds that will play a role in the analysis of this paper are the so-called proportional lower bounds, which assign to each agent a fixed portion of the proportional allocation. Formally, for each $\alpha \in(0,1), \rho^{\alpha}(N, c, E)=\frac{\alpha E}{C} c$. The corresponding (proportional) upper bounds are easily obtained: $\hat{\rho}^{\alpha}(N, c, E)=\frac{(1-\alpha) C+\alpha E}{C} c$.

It might well be the case that a baseline is neither a lower bound nor an upper bound. Instances are those baselines that do not depend on the available amount. A focal example will be the case in which baselines are a given percentage of claims. Formally, for each $\theta \in(0,1)$, $\theta(N, c, E)=\theta c$, for all $(N, c, E) \in \mathcal{D}$.

\section{Composition Operators}

We now introduce the concept of a composition operator. More precisely, for a given baseline $b$, the composition operator $O^{b}$ is the operator assigning to each rule $R$ the rule $R^{b}$ arising from composing the tentative allocation of $b$ with the allocation that $R$ proposes for the revised problem. Formally,

$R^{b}(N, c, E)= \begin{cases}R(N, b(N, c, E), E) & \text { if } E \leq \sum_{i} b_{i}(N, c, E) \\ b(N, c, E)+R\left(N, c-b(N, c, E), E-\sum_{i} b_{i}(N, c, E)\right) & \text { if } E \geq \sum_{i} b_{i}(N, c, E)\end{cases}$

A straightforward consequence of (1) is that $R^{b}$ yields allocations satisfying $x_{i} \leq b_{i}(N, c, E)$ for all $i \in N$ if and only if $E \leq \sum_{i} b_{i}(N, c, E)$, and $x_{i} \geq b_{i}(N, c, E)$ for all $i \in N$ if and only if $E \geq \sum b_{i}(N, c, E)$. In words, $R^{b}$ imposes a rationing of the same sort for each individual and the whole society according to the profile of baselines.

It can be argued that $R^{b}$ rations agents relative to the feasible or unfeasible baselines in the spirit of composition up and down respectively. More precisely, if $b$ is a feasible baseline (i.e., a lower bound), then

$$
R^{b}(N, c, E)=b(N, c, E)+R\left(N, c-b(N, c, E), E-\sum_{i \in N} b_{i}(N, c, E)\right)
$$

as in the spirit of composition up, where $R^{b}$ first allocates $b$ and then allocates the residual amount using the rule $R$ with respect to residual claims $c-b$.

If $b$ is an unfeasible baseline (i.e., an upper bound), then

$$
R^{b}(N, c, E)=R(N, b(N, c, E), E)
$$

as in the spirit of composition down, where $R^{b}$ allocates $E$ by using the rule $R$ with respect to the baseline itself. 
The following lemma, whose straightforward proof we omit, shows that the minimal rights operator and the claims truncation operator are specific instances of composition operators.

Lemma 1 The following statements hold:

- If $b(N, c, E)=m(N, c, E)$ for all $(N, c, E) \in \mathcal{D}$, then $O^{b} \equiv O^{m}$.

- If $b(N, c, E)=t(N, c, E)$ for all $(N, c, E) \in \mathcal{D}$, then $O^{b} \equiv O^{t}$.

As mentioned above, a natural class of baselines arises when baselines profiles are assumed to be a given percentage of claims, i.e., for some $\theta \in(0,1), b(N, E, c)=\theta c$, for all $(N, c, E) \in \mathcal{D}$. It turns out that the proportional rule is a fixed point for each of the corresponding composition operators $\left\{O^{\theta}\right\}_{\theta \in(0,1)}$. Formally, $O^{\theta}(P)=P$, for all $\theta \in(0,1) .{ }^{5}$ As for the specific case in which $\theta=1 / 2$, which could be interpreted as a psychological threshold, the other two classical rules (namely, the constrained equal awards and the constrained equal losses rules) also provide interesting outcomes. More precisely, it is straightforward to show that $O^{1 / 2}(A)$ would precisely be the so-called Piniles rule (e.g., Thomson, 2003), whereas $O^{1 / 2}(L)$ would be its dual rule.

\section{Relating operators}

\subsection{Duality relationships}

We now relate some of the composition operators described above. Note first that, as the duality operator is an involution, then $R$ is the dual of $S$ if and only if $S$ is the dual of $R$. Hence, if $R$ is the dual of $S$ we can refer to $R$ and $S$ as dual rules.

We now define the concept of a dual baseline. Formally, for a given baseline $b$, we define its dual $\widehat{b}$ by

$$
\widehat{b}(N, c, E)=c-b(N, c, C-E)
$$

In words, as it happens with rules, the baseline $\widehat{b}$ allocates awards in the same way as baseline $b$ allocates losses. It is straightforward to see that the dual of a lower bound is an upper bound and vice versa. Note also that $E \geq \sum b_{i}(N, c, E)$ is equivalent to saying that $C-E \leq$ $\sum \widehat{b}_{i}(N, c, C-E)$. Then, we have the following result:

Theorem 1 Let $R$ and $S$ be dual rules. Then $R^{b}$ and $S^{\widehat{b}}$ are dual rules.

\footnotetext{
${ }^{5}$ That is also the case for the proportional bounds operators $\left\{O^{\rho^{\alpha}}\right\}_{\alpha \in(0,1)}$ and $\left\{O^{\hat{\rho}^{\alpha}}\right\}_{\alpha \in(0,1)}$.
} 
Proof. We need to show that for each $(N, c, E), R^{b}(N, c, E)=c-S^{\widehat{b}}(N, c, C-E)$. We distinguish two cases.

Case 1: $E \leq \sum_{i \in N} b_{i}(N, c, E)$.

In this case, $R^{b}(N, c, E)=R(N, b(N, c, E), E)$ and

$$
\begin{aligned}
c-S^{\widehat{b}}(N, c, C-E) & =c-\widehat{b}(N, c, C-E)-S\left(N, c-\widehat{b}(N, c, C-E), C-E-\sum_{i \in N} \widehat{b}_{i}(N, c, C-E)\right) \\
& =b(N, c, E)-S\left(N, b(N, c, E), \sum_{i \in N} b_{i}(N, c, E)-E\right) .
\end{aligned}
$$

Now, as $R$ and $S$ are dual rules, it follows that

$$
R(N, b(N, c, E), E)=b(N, c, E)-S\left(N, b(N, c, E), \sum_{i \in N} b_{i}(N, c, E)-E\right)
$$

Thus, $R^{b}(N, c, E)=c-S^{\widehat{b}}(N, c, C-E)$, as desired.

Case 2: $E \geq \sum_{i \in N} b_{i}(N, c, E)$.

In this case,

$$
R^{b}(N, c, E)=b(N, c, E)+R\left(N, c-b(N, c, E), E-\sum_{i \in N} b_{i}(N, c, E)\right)
$$

and

$$
c-S^{\widehat{b}}(N, c, C-E)=c-S(N, \widehat{b}(N, c, C-E), C-E)=c-S(N, c-b(N, c, E), C-E)
$$

Now, as $R$ and $S$ are dual rules, it follows that

$$
R\left(N, c-b(N, c, E), E-\sum_{i \in N} b_{i}(N, c, E)\right)=c-b(N, c, E)-S(N, c-b(N, c, E), C-E)
$$

from where the desired equality follows.

Theorem 1 says, in particular, that if $R$ is a self-dual rule, then $R^{b}$ and $R^{\widehat{b}}$ are dual rules. Somewhat related, we also have that if $b$ is a given baseline, $S$ is a rule such that $S^{b}$ is self-dual, and $R$ is the dual rule of $S$, then $R^{\hat{b}} \equiv S^{b}$.

It is straightforward to see that the truncated claims application and the minimal rights application are dual baselines, i.e., $\widehat{t}=m$ and $\widehat{m}=t$. We then have the following corollary, which corresponds to Theorems 1 and 5 in Thomson and Yeh (2008).

Corollary 1 Let $R$ and $S$ be two dual rules. Then, $R^{m}$ and $S^{t}$ are dual rules. 
Other similar corollaries can also be obtained as straightforward consequences of the above theorem.

Corollary 2 Let $R$ and $S$ be two dual rules. Then,

- $R^{\mu}$ and $S^{\hat{\mu}}$ are dual rules.

- $R^{\rho^{\alpha}}$ and $S^{\hat{\rho}^{\alpha}}$ are dual rules.

- $R^{\theta}$ and $S^{1-\theta}$ are dual rules. ${ }^{6}$

\subsection{Commutative relationships}

We now turn to explore how composition operators behave when they are applied sequentially. More precisely, our next result shows that the composition operators corresponding to two dual baselines commute, provided they satisfy an additional condition. Formally, let $b$ be a lower bound. We say that $b$ is duality stable if

$$
b(N, \widehat{b}(N, c, E), E)=b(N, c, E)
$$

for all $(N, c, E) \in \mathcal{D}$. Then, we have the following:

Theorem 2 If a lower bound is duality stable then the composition operator $O^{b}$ commutes with the composition operator $O^{\widehat{b}}$, i.e., for each rule $R$,

$$
O^{\widehat{b}}\left(O^{b}(R(N, c, E))\right)=O^{b}\left(O^{\widehat{b}}(R(N, c, E))\right) .
$$

Proof. Let $R$ be a given rule and $(N, c, E) \in \mathcal{D}$ a given problem.

On the one hand, we have that

$$
\begin{aligned}
O^{\widehat{b}}\left(O^{b}(R(N, c, E))\right) & =R^{b}(N, \widehat{b}(N, c, E), E) \\
& =b(N, \widehat{b}(N, c, E), E) \\
& +R\left(N, \widehat{b}(N, c, E)-b(N, \widehat{b}(N, c, E), E), E-\sum_{i \in N} b_{i}(N, \widehat{b}(N, c, E), E)\right) .
\end{aligned}
$$

On the other hand, we have that

$$
\begin{aligned}
O^{b}\left(O^{\widehat{b}}(R(N, c, E))\right) & =b(N, c, E)+R^{\widehat{b}}\left(N, c-b(N, c, E), E-\sum_{i \in N} b_{i}(N, c, E)\right) \\
& =b(N, c, E) \\
& +R\left(N, \widehat{b}\left(N, c-b(N, c, E), E-\sum_{i \in N} b_{i}(N, c, E)\right), E-\sum_{i \in N} b_{i}(N, c, E)\right) .
\end{aligned}
$$

\footnotetext{
${ }^{6}$ In particular, if $R$ is a self-dual rule then $R^{1 / 2}$ is self-dual too.
} 
It follows from (2) that

$$
\begin{aligned}
\widehat{b}\left(N, c-b(N, c, E), E-\sum_{i \in N} b_{i}(N, c, E)\right) & =c-b(N, c, E)-b(N, c-b(N, c, E), C-E) \\
& =c-b(N, c, E)-b(N, \widehat{b}(N, c, C-E), C-E) \\
& =c-b(N, c, E)-b(N, c, C-E) \\
& =\widehat{b}(N, c, E)-b(N, c, E) .
\end{aligned}
$$

Thus, by dual stability, both (3) and (4) are equal to

$$
b(N, c, E)+R\left(N, \widehat{b}(N, c, E)-b(N, c, E), E-\sum_{i \in N} b_{i}(N, c, E)\right),
$$

which concludes the proof.

It is not difficult to show that the minimal rights lower bound is duality stable. Consequently, we have the following corollary, which corresponds to Theorem 3 in Thomson and Yeh (2008).

\section{Corollary $3 O^{m}$ and $O^{t}$ commute.}

The proportional lower bounds are duality stable too. Consequently, we have that $O^{\rho^{\alpha}}$ and $O^{\hat{\rho}^{\alpha}}$ commute, for any $\alpha \in(0,1)$. The lower bound $\mu$, however, is not duality stable and, actually, it is not difficult to show that $O^{\mu}$ and $O^{\hat{\mu}}$ do not commute. The operators $O^{\theta}$ and $O^{1-\theta}$ do not commute either, for any $\theta \in(0,1)$.

Theorem 2 can be further extended in the two-agent case for rules satisfying a mild notion of impartiality stating that agents with equal claims are awarded equally. In order to present that result, let us refer first to a focal principle in the two-agent case, known as concedeand-divide, whose motivation can be traced back to the Talmud. It amounts to solve twoagent problems upon conceding each agent a portion of the available amount and dividing the remainder equally. A point in case for such concessions are the minimal rights described above, but any other lower bound could be considered too. Formally, for any lower bound $b$, the so-called generalized concede-and-divide rule, $C D^{b}$, selects, for each two-agent problem $\left(\{i, j\},\left(c_{i}, c_{j}\right), E\right)$, the allocation

$$
\left(b_{i}\left(\{i, j\},\left(c_{i}, c_{j}\right), E\right)+\frac{E-B\left(\{i, j\},\left(c_{i}, c_{j}\right), E\right)}{2}, b_{j}\left(\{i, j\},\left(c_{i}, c_{j}\right), E\right)+\frac{E-B\left(\{i, j\},\left(c_{i}, c_{j}\right), E\right)}{2}\right),
$$

where $B\left(\{i, j\},\left(c_{i}, c_{j}\right), E\right)=b_{i}\left(\{i, j\},\left(c_{i}, c_{j}\right), E\right)+b_{j}\left(\{i, j\},\left(c_{i}, c_{j}\right), E\right)$. The so-called concedeand-divide rule, $C D$, which corresponds to the two-agent version of the Talmud rule, is obtained when $b=m$. 
We now introduce an additional condition for lower bounds. Formally, let $b$ be a lower bound. We say that $b$ satisfies constant duality gap if, for all $(N, c, E) \in \mathcal{D}$,

$$
\widehat{b}_{i}(N, c, E)-b_{i}(N, c, E)=\widehat{b}_{j}(N, c, E)-b_{j}(N, c, E)
$$

for all $i, j \in N$.

The following result strengthens Theorem 2 in the two-agent case.

Theorem 3 If $R$ is a two-agent rule satisfying equal treatment of equals, and $b$ is a lower bound satisfying dual stability and constant duality gap in the two-agent case, then $O^{b}\left(O^{\hat{b}}(R)\right)=$ $O^{\hat{b}}\left(O^{b}(R)\right)=C D^{b}$.

Proof. Let $R$ be a two-agent rule satisfying equal treatment of equals, and let $b$ be a lower bound satisfying dual stability and constant duality gap in the two-agent case. Let $\left(\{i, j\},\left(c_{i}, c_{j}\right), E\right) \in \mathcal{D}$ be a two-agent problem. Then, by the proof of Theorem 2,

$$
\begin{aligned}
& O^{\widehat{b}}\left(O^{b}\left(R\left(\{i, j\},\left(c_{i}, c_{j}\right), E\right)\right)\right)=O^{b}\left(O^{\widehat{b}}\left(R\left(\{i, j\},\left(c_{i}, c_{j}\right), E\right)\right)\right)= \\
& b\left(\{i, j\},\left(c_{i}, c_{j}\right), E\right)+R\left(\{i, j\}, \widehat{b}\left(\{i, j\},\left(c_{i}, c_{j}\right), E\right)-b\left(\{i, j\},\left(c_{i}, c_{j}\right), E\right), E-B\left(\{i, j\},\left(c_{i}, c_{j}\right), E\right)\right),
\end{aligned}
$$

where $B\left(\{i, j\},\left(c_{i}, c_{j}\right), E\right)=b_{i}\left(\{i, j\},\left(c_{i}, c_{j}\right), E\right)+b_{j}\left(\{i, j\},\left(c_{i}, c_{j}\right), E\right)$. By constant duality gap (of $b$ ) and equal treatment of equals (of $R$ ) the result follows. ${ }^{7}$

It is straightforward to show that the minimal rights lower bound satisfies constant duality gap in the two-agent case. Consequently, we have the following corollary, which corresponds to Theorem 2 in Thomson and Yeh (2008).

Corollary 4 If $R$ is a two-agent rule satisfying equal treatment of equals, then $O^{m}\left(O^{t}(R)\right)=$ $O^{t}\left(O^{m}(R)\right)=C D$.

\subsection{Fixed points and recursive iterations}

Another interesting issue related to operators is to explore the rules that remain unchanged when applied to them. A similar question has been recently addressed by Dominguez (2010) in the specific case of lower bounds. More precisely, Dominguez (2010) shows that if a lower bound $b$ is continuous and satisfies positivity (i.e., for all $(N, c, E) \in \mathcal{D}, b_{i}(N, c, E) \geq 0$ for all

\footnotetext{
${ }^{7}$ Note that the result could be extended to the general case of $n$ claimants upon imposing constant duality gap for that general case, and obtaining the rule that allocates the lower bound first and then divides the remainder equally among all agents.
} 
$i \in N$, with some strict inequality), then there is a unique rule satisfying invariance under the assignment of such lower bound (in the parlance of our paper, the composition operator $O^{b}$ has a unique fixed point, i.e., there is a unique $S$ such that $S^{b}=S$ ). Furthermore, such a rule is obtained upon applying recursively the lower bound. ${ }^{8}$ Thanks to Theorem 1 , we can extend such a result for upper bounds and baselines in general. We say that an upper bound satisfies strictness if for all $(N, c, E) \in \mathcal{D}, b_{i}(N, c, E) \leq c_{i}$ for all $i \in N$, with some strict inequality. It is straightforward to show that a lower bound satisfies positivity if and only if its dual upper bound satisfies strictness. The next result summarizes our findings in this section. ${ }^{9}$

\section{Theorem 4 The following statements hold:}

- If a continuous lower bound $b$ satisfies positivity then there is a unique fixed point of $O^{b}$. Such a fixed point is the rule obtained upon applying recursively the lower bound $b$.

- If a continuous upper bound $b$ satisfies strictness then there is a unique fixed point of $O^{b}$. Such a fixed point is the rule obtained upon applying recursively the upper bound $b$, and it corresponds to the dual rule of the unique fixed point of $O^{\hat{b}}$.

- If a continuous baseline b satisfies strictness and positivity then there is a unique fixed point of $O^{b}$. Such a fixed point is the rule obtained upon applying recursively the baseline $b$.

Proof. As mentioned above, the first statement is shown by Dominguez (2010). As for the second statement, let $b$ be a continuous upper bound satisfying strictness. Then, $\hat{b}$ is a continuous lower bound that satisfies positivity. By the first statement, we know that there is a unique rule $S$, such that $S^{\hat{b}}=S$ (and, moreover, $S$ is the rule that is obtained by applying the bound iteratively). Then, $O^{d}\left(S^{\hat{b}}\right)=O^{d}(S)$. Let $R=O^{d}(S)$. As $R$ and $S$ are dual rules, then, by Theorem 1, so are $S^{\hat{b}}$ and $R^{b}$. Thus, $R^{b}=O^{d}\left(S^{\hat{b}}\right)=O^{d}(S)=R$, which shows that $R$ is indeed the unique fixed point of $O^{b}$. It also follows, from duality, that $R$ is the rule obtained upon applying recursively the upper bound $b$. This proves the second statement. The third one follows from the first two statements. More precisely, positivity guarantees that the residual amount to be allocated decreases for those steps in which the recursive iteration yields a

\footnotetext{
${ }^{8}$ It is worth mentioning that if positivity is dropped then the result does not hold, and the corresponding composition operator may well have many fixed points. For instance, all rules within the TAL-family (e.g., Moreno-Ternero and Villar, 2006) from the constrained equal losses rule to the Talmud rule are fixed points of $O^{m}$.

${ }^{9}$ Statement 1 is due to Dominguez (2010).
} 
feasible vector, as in the case of lower bounds; strictness guarantees that claims are reduced for those steps in which the recursive iteration yields an unfeasible vector, as in the case of upper bounds. Continuity concludes.

It is worth mentioning that, as with the case of positivity, strictness is necessary to guarantee the uniqueness stated in the previous statements. ${ }^{10}$ Note that the upper bound $\hat{\mu}$ satisfies strictness. The unique fixed point of its corresponding composition operator is hence the dual rule of the so-called recursive rule, introduced and analyzed by Dominguez and Thomson (2006). Proportional lower (upper) bounds satisfy positivity (strictness). As mentioned above, the proportional rule is a fixed point of the corresponding operators. Theorem 4 shows uniqueness. As for the operators $\left\{O^{\theta}\right\}_{\theta \in(0,1)}$, Theorem 4 also shows that they each have a unique fixed point.

\section{Preservation of axioms under composition operators}

An axiom is said to be preserved under an operator if any rule that satisfies the axiom is mapped by the operator into a rule that also satisfies the axiom. The literature has provided a wide variety of axioms for rules reflecting ethical or operational principles. Here we shall concentrate on those formalizing the principles of impartiality, priority, and solidarity, which have a long tradition in the theory of justice (e.g., Moreno-Ternero and Roemer, 2006), but also some operational (independence, strategic, and procedural) properties widely used in the literature on the problem of adjudicating conflicting claims (e.g., Thomson, 2003).

Impartiality refers to the fact that ethically irrelevant information is excluded from the allocation process. In this context, it is modeled by the axiom of Equal Treatment of Equals, which requires allotting equal amounts to those agents with equal claims. Formally, a rule $R$ satisfies equal treatment of equals if, for all $(N, c, E) \in \mathcal{D}$, and all $i, j \in N$, we have $R_{i}(N, c, E)=R_{j}(N, c, E)$, whenever $c_{i}=c_{j}$. A strengthening of equal treatment of equals is the requirement that the identity of agents should not matter. The chosen awards vector should depend only on the list of claims, not on who holds them. Formally, let $\Pi^{N}$ denote the class of bijections from $N$ into itself. A rule $R$ satisfies Anonymity if, for all $(N, c, E) \in \mathcal{D}$, $\pi \in \Pi^{N}$, and $i \in N, R_{\pi(i)}\left(N,\left(c_{\pi(i)}\right)_{i \in N}, E\right)=R_{i}(N, c, E)$.

\footnotetext{
${ }^{10}$ If strictness is dropped then the corresponding composition operator may well have many fixed points. For instance, all rules within the TAL-family (e.g., Moreno-Ternero and Villar, 2006) from the Talmud rule to the constrained equal awards rule are fixed points of $O^{t}$.
} 
The principle of priority requires imposing a positive discrimination (albeit only to a certain extent) towards individuals with higher needs. In this context, needs are reflected by claims and as such, priority is modeled by the axiom of Order Preservation, which says that agents with larger claims receive larger awards but face larger losses too. That is, $c_{i} \geq c_{j}$ implies that $R_{i}(N, c, E) \geq R_{j}(N, c, E)$ and $c_{i}-R_{i}(N, c, E) \geq c_{j}-R_{j}(N, c, E)$, for all $(N, c, E) \in \mathcal{D}$, all $i, j \in N$.

The principle of solidarity, with a long tradition in the axiomatic literature, can be modeled in various related ways. Resource Monotonicity says that when there is more to be divided, other things being equal, nobody should lose. Formally, a rule $R$ is resource monotonic if, for each $(N, c, E) \in \mathcal{D}$ and $\left(N, c, E^{\prime}\right) \in \mathcal{D}$ such that $E \leq E^{\prime}$, then $R(N, c, E) \leq R\left(N, c, E^{\prime}\right)$. Claims Monotonicity says that if an agent's claim increases, ceteris paribus, she should receive at least as much as she did initially. Formally, a rule $R$ is claims monotonic if, for all $(N, c, E) \in \mathcal{D}$ and all $i \in N, c_{i} \leq c_{i}^{\prime}$ implies $R_{i}\left(N,\left(c_{i}, c_{N \backslash\{i\}}\right), E\right) \leq R_{i}\left(N,\left(c_{i}^{\prime}, c_{N \backslash\{i\}}\right), E\right)$. The dual property says that if an agent's claim and the amount to divide increase by the same amount, the agent's award should increase by at most that amount. Formally, a rule satisfies Linked Monotonicity if, for all $(N, c, E) \in \mathcal{D}$ and $i \in N, R_{i}\left(N,\left(c_{i}+\varepsilon, c_{N \backslash\{i\}}\right), E+\varepsilon\right) \leq R_{i}(N, c, E)+\varepsilon{ }^{11}$

Population monotonicity is a relevant solidarity property in the context of a variable population. It says that if new claimants arrive, each claimant initially present should receive at most as much as he did initially. Equivalently, if some claimants leave but there still is not enough to honor all of the remaining claims, each remaining claimant should receive at least as much as he did initially. Formally, $R$ is population monotonic if for all $(N, c, E) \in \mathcal{D}$ and $\left(N^{\prime}, c^{\prime}, E\right) \in \mathcal{D}$ such that $N \subseteq N^{\prime}$ and $c_{N}^{\prime}=c$, then $R_{i}\left(N^{\prime}, c^{\prime}, E\right) \leq R_{i}(N, c, E)$, for all $i \in N$. The dual of population monotonicity says that if new claimants arrive and the amount to divide increases by the sum of their claims, then each claimant initially present should receive at least as much as he did initially. Formally, a rule $R$ satisfies Resource-and-Population Monotonicity if for all $(N, c, E) \in \mathcal{D}$ and $\left(N^{\prime}, c^{\prime}, E\right) \in \mathcal{D}$ such that $N \subseteq N^{\prime}$ and $c_{N}^{\prime}=c$, then $R_{i}(N, c, E) \leq R_{i}\left(N^{\prime}, c^{\prime}, E+\sum_{N^{\prime} \backslash N} c_{j}\right), i \in N$.

The next axiom also amounts to simultaneous changes in the amount available and the population. It says that the arrival of new agents should affect all the incumbent agents in the same direction. In other words, agents cannot benefit from a change (either in the

\footnotetext{
${ }^{11}$ For any given property $\alpha, \alpha^{*}$ is the dual property of $\alpha$ if for each rule $R, R$ satisfies $\alpha$ if and only if its dual rule $R^{*}$ satisfies $\alpha^{*}$. A property is said to be self-dual if it coincides with its dual. Equal treatment of equals, order preservation and resource monotonicity are instances of self-dual properties.
} 
available wealth or in the number of agents) if someone else suffers from it. Formally, a rule $R$ satisfies Resource-and-Population Uniformity if for all $(N, c, E) \in \mathcal{D}$ and $\left(N^{\prime}, c^{\prime}, E^{\prime}\right) \in \mathcal{D}$ such that $N \subseteq N^{\prime}$ and $c_{N}^{\prime}=c$, then, either $R_{i}\left(N^{\prime}, c^{\prime}, E^{\prime}\right) \leq R_{i}(N, c, E)$, for all $i \in N$, or $R_{i}\left(N^{\prime}, c^{\prime}, E^{\prime}\right) \geq R_{i}(N, c, E)$, for all $i \in N$. It is straightforward to show that this axiom implies resource monotonicity. As a matter of fact, it also satisfies the following axiom that relates the solution of a given problem to the solutions of the subproblems that appear when we consider a subgroup of agents as a new population and the amounts gathered in the original problem as the available amount to be distributed. Consistency requires that the application of the rule to each subproblem produces precisely the allocation that the subgroup obtained in the original problem. ${ }^{12}$ More formally: A rule $R$ is consistent if, for all $(N, c, E) \in \mathcal{D}$, all $M \subset N$, and all $i \in M$, we have $R_{i}(N, c, E)=R_{i}\left(M, c_{M}, E_{M}\right)$, where $E_{M}=\sum_{i \in M} R_{i}(N, c, E)$. It turns out that consistency and resource monotonicity together imply resource-and-population uniformity.

We now move to a group of operational properties.

We say that a rule $R$ satisfies Scale Invariance when if claims and amount available are multiplied by the same positive number, then so should all awards. Formally, for all $(N, c, E) \in$ $\mathcal{D}$ and $\lambda \in \mathbb{R}_{+}, R(N, \lambda c, \lambda E)=\lambda R(N, c, E)$.

We continue stating formally the two composition properties that were motivated at the introduction. A rule $R$ satisfies Composition $U p$ if, for all $(N, c, E) \in \mathcal{D}$ and all $E_{1}, E_{2} \in \mathbb{R}_{++}$such that $E_{1}+E_{2}=E, R(N, c, E)=R\left(N, c, E_{1}\right)+R\left(N, c-R\left(N, c, E_{1}\right), E_{2}\right)$. It satisfies Composition Down if, for all $(N, c, E) \in \mathcal{D}$ and all $E^{\prime}>E$, we have $R(N, c, E)=R\left(N, R\left(N, c, E^{\prime}\right), E\right)$.

Somewhat related, we state the two properties that motivated the definition of the operators $O^{m}$ and $O^{t}$. A rule $R$ satisfies Minimal Rights First if $R(N, c, E)=m(N, c, E)+R(N, c-$ $\left.m(N, c, E), E-\sum_{i} m_{i}(N, c, E)\right)=R^{m}(N, c, E)$, for all $(N, c, E) \in \mathcal{D}$, and Claims Truncation Invariance if $R(N, c, E)=R(N, t(N, c, E), E)=R^{t}(N, c, E)$, for all $(N, c, E) \in \mathcal{D}$. These properties can be easily generalized to arbitrary lower and upper bounds, respectively. More precisely, for any lower bound $b$, we say that a rule satisfies $b$-first if $R(N, c, E)=b(N, c, E)+$ $R\left(N, c-b(N, c, E), E-\sum_{i} b_{i}(N, c, E)\right)=R^{b}(N, c, E)$, for all $(N, c, E) \in \mathcal{D}$. Similarly, for any upper bound $b$, we say that a rule satisfies b-invariance, if $R(N, c, E)=R(N, b(N, c, E), E)=$ $R^{b}(N, c, E)$, for all $(N, c, E) \in \mathcal{D} .^{13}$ It is not difficult to show that if $\hat{b}$ is the dual baseline of a

\footnotetext{
${ }^{12}$ See Thomson (1996) for an excellent survey of the many applications that have been made on the idea of consistency.

${ }^{13}$ Using a different parlance, a rule satisfies $b$-first if $b$ is a lower bound and the rule is a fixed point of $O^{b}$,
} 
given lower bound $b$ then $b$-first and $\hat{b}$-invariance are dual properties.

\subsection{Direct preservation}

Our next result helps to identify the preservation of some axioms.

Theorem 5 A property is preserved under the composition operator $O^{b}$ if and only if its dual property is preserved under the composition operator $O^{\widehat{b}}$.

Proof. Let $\mathcal{P}$ be a property that is preserved under $O^{b}, \mathcal{Q}$ its dual, and let $R$ be a rule satisfying $\mathcal{Q}$. We need to show that $R^{\widehat{b}}$ satisfies $\mathcal{Q}$. As $\mathcal{P}$ is dual to $\mathcal{Q}, S$, the dual rule of $R$, satisfies $\mathcal{P}$. As $\mathcal{P}$ is preserved under $O^{b}$, it follows that $S^{b}$ satisfies $\mathcal{P}$. By Theorem $1, S^{b}=R^{\widehat{b}}$, which concludes the proof of the "if" statement. The proof of the "only if" part goes along the same lines.

The next corollary summarizes some straightforward consequences of the above theorem. Its first statement corresponds to Theorem 4 in Thomson and Yeh (2008).

Corollary 5 The following statements hold:

- A property is preserved under $O^{m}$ if and only if its dual property is preserved under $O^{t}$.

- A property is preserved under $O^{\mu}$ if and only if its dual property is preserved under $O^{\hat{\mu}}$.

- A property is preserved under $O^{\rho^{\alpha}}$ if and only if its dual property is preserved under $O^{\hat{\rho}^{\alpha}}$.

- A property is preserved under $O^{\theta}$ if and only if its dual property is preserved under $O^{1-\theta}$.

For arbitrary baseline profiles it is not difficult to show that almost none of the above axioms are preserved by the composition operators. A notable exception are the so-called independence properties that generalize the properties of minimal rights first and claims truncation invariance described above.

Proposition 1 The following statements hold:

- If $b$ is a lower bound then $O^{b}$ preserves b-first.

- If $b$ is an upper bound then $O^{b}$ preserves b-invariance.

and b-invariance if $b$ is an upper bound and the rule is a fixed point of $O^{b}$. 
Proof. By Theorem 5, we only need to prove one statement. Let $b$ be an upper bound, $R$ a rule satisfying $b$-invariance, and $S=O^{b}(R)=R^{b}$. For any $(N, c, E) \in \mathcal{D}, S^{b}(N, c, E)=$ $S(N, b(N, c, E), E)=R^{b}(N, b(N, c, E), E)$. As $R$ satisfies $b$-invariance, it follows that

$$
R^{b}(N, b(N, c, E), E)=R(N, b(N, c, E), E)=R^{b}(N, c, E)=S(N, c, E)
$$

Altogether, we have that $S^{b}(N, c, E)=S(N, c, E)$, as desired.

As a consequence of the above proposition we have that $O^{t}$ preserves invariance under claims truncation, and that $O^{m}$ preserves minimal rights first, as proved by Thomson and Yeh (2008) directly.

\subsection{Consequent preservation}

As mentioned above, most of the usual axioms are not preserved by the composition operators. It turns out, however, that many axioms are consequently preserved. By consequent preservation we mean that if a rule $R$ satisfies a property $\mathcal{P}$, and the baseline does that too, then $R^{b}$ also satisfies this property.

Proposition 2 Equal treatment of equals, anonymity, order preservation and scale invariance are consequently preserved.

\section{Proof.}

Let us start with equal treatment of equals. A baseline $b$ satisfies equal treatment of equals if for all $(N, c, E) \in \mathcal{D}$, and $i, j \in N$ such that $c_{i}=c_{j}$ then $b_{i}(N, c, E)=b_{j}(N, c, E)$. Let $R$ and $b$ be a rule and a baseline, respectively, satisfying equal treatment of equals. Let $(N, c, E) \in$ $\mathcal{D}$ be given and let $i, j \in N$ be such that $c_{i}=c_{j}$. Then, $b_{i}(N, c, E)=b_{j}(N, c, E)$ and $c_{i}-b_{i}(N, c, E)=c_{j}-b_{j}(N, c, E)$ and therefore, $R_{i}^{b}(N, c, E)=R_{j}^{b}(N, c, E)$.

A baseline $b$ is anonymous if for all $(N, c, E) \in \mathcal{D}, \pi \in \Pi^{N}$, and $i \in N, b_{\pi(i)}\left(N,\left(c_{\pi(i)}\right)_{i \in N}, E\right)=$ $b_{i}(N, c, E)$. Let $R$ and $b$ be an anonymous rule and baseline, respectively. Let $(N, c, E) \in \mathcal{D}$ be given and let also $\pi \in \Pi^{N}$ and $i \in N$ be given. Then, if $E \leq \sum_{i} b_{i}(N, c, E), R_{\pi(i)}^{b}\left(N,\left(c_{\pi(i)}\right)_{i \in N}, E\right)=$ $R_{\pi(i)}\left(N, b\left(N,\left(c_{\pi(i)}\right)_{i \in N}, E\right), E\right)=R_{\pi(i)}\left(N,\left(b_{\pi(i)}\left(N,\left(c_{\pi(i)}\right)_{i \in N}, E\right)\right)_{i \in N}, E\right)=R_{i}(N, b(N, c, E), E)=$ $R_{i}^{b}(N, c, E)$. Similarly, if $E \geq \sum_{i} b_{i}(N, c, E)$, then $R_{\pi(i)}^{b}\left(N,\left(c_{\pi(i)}\right)_{i \in N}, E\right)=b_{\pi(i)}\left(N,\left(c_{\pi(i)}\right)_{i \in N}, E\right)+$ $R_{\pi(i)}\left(N,\left(c_{\pi(i)}-\left(b_{i}\left(N,\left(c_{\pi(i)}\right)_{i \in N}, E\right)\right)_{i \in N}, E-\sum_{i} b_{i}\left(N,\left(c_{\pi(i)}\right)_{i \in N}, E\right)\right)=b_{i}(N, c, E)\right)+R_{i}(N,(c-$ $\left.\left.b(N, c, E), E-\sum_{i} b_{i}(N, c, E)\right)=R_{i}^{b}(N, c, E)\right)$, as desired.

We now move to order preservation. A baseline $b$ is order preserving if for all $(N, c, E) \in$ $\mathcal{D}$, and $i, j \in N$ such that $c_{i}<c_{j}$ then $b_{i}(N, c, E) \leq b_{j}(N, c, E)$ and $c_{i}-b_{i}(N, c, E) \leq$ 
$c_{j}-b_{j}(N, c, E)$. Let $R$ and $b$ be an order-preserving rule and baseline, respectively. Let $(N, c, E) \in \mathcal{D}$ be given and let $i, j \in N$ be such that $c_{i}<c_{j}$. Then, $b_{i}(N, c, E) \leq b_{j}(N, c, E)$ and $c_{i}-b_{i}(N, c, E) \leq c_{j}-b_{j}(N, c, E)$. As $R$ is order preserving, it follows that $R_{i}(N, b, E) \leq$ $R_{j}(N, b, E)$ if $E \leq \sum_{i} b_{i}(N, c, E)$ and that $b_{i}(N, c, E)+R_{i}\left(N, c-b, E-\sum_{i} b_{i}(N, c, E)\right) \leq$ $b_{j}(N, c, E)+R_{j}\left(N, c-b, E-\sum_{i} b_{i}(N, c, E)\right)$ if $E \geq \sum_{i} b_{i}(N, c, E)$. Then, $R_{i}^{b}(N, c, E) \leq$ $R_{j}^{b}(N, c, E)$. Similarly, as $R$ is order preserving, it follows that $b_{i}(N, c, E)-R_{i}(N, b, E) \leq$ $b_{j}(N, c, E)-R_{j}(N, b, E)$ if $E \leq \sum_{i} b_{i}(N, c, E)$. As $b$ is order preserving, this implies that $c_{i}-R_{i}(N, b, E) \leq c_{j}-R_{j}(N, b, E)$ if $E \leq \sum_{i} b_{i}(N, c, E)$. Finally, as $R$ and $b$ are order preserving, it follows that $c_{i}-b_{i}(N, c, E)-R_{i}\left(N, c-b, E-\sum_{i} b_{i}(N, c, E)\right) \leq c_{j}-b_{j}(N, c, E)-$ $R_{j}\left(N, c-b, E-\sum_{i} b_{i}(N, c, E)\right)$ if $E \geq \sum_{i} b_{i}(N, c, E)$. Thus, $c_{i}-R_{i}^{b}(N, c, E) \leq c_{j}-R_{j}^{b}(N, c, E)$, which concludes the proof.

Finally, a baseline $b$ is scale invariant if for all $(N, c, E) \in \mathcal{D}$, and $\lambda>0, b(N, \lambda c, \lambda E)=$ $\lambda b(N, c, E)$. Let $R$ and $b$ be a scale-invariant rule and baseline, respectively. Let $(N, c, E) \in \mathcal{D}$ be given and let $\lambda>0$ be given. Then, if $E \leq \sum_{i} b_{i}(N, c, E), R^{b}(N, \lambda c, \lambda E)=R(N, b(N, \lambda c, \lambda E), \lambda E)=$ $R(N, \lambda b(N, c, E), \lambda E)=\lambda R(N, b(N, c, E), E)=\lambda R^{b}(N, c, E)$. Similarly, if $E \geq \sum_{i} b_{i}(N, c, E)$, then $R^{b}(N, \lambda c, \lambda E)=b(N, \lambda c, \lambda E)+R(N, \lambda c-b(N, \lambda c, \lambda E), \lambda E)=\lambda b(N, c, E)+\lambda R(N, b(N, c, E), E)=$ $\lambda R^{b}(N, c, E)$, as desired.

The following corollary, whose content is shown directly by Thomson and Yeh (2008), is a straightforward consequence of the above proposition.

Corollary 6 Equal treatment of equals, anonymity, order preservation, and scale invariance are preserved by $O^{m}$ and $O^{t}$.

\subsection{Assisted preservation}

We now move to a set of properties that require additional conditions on baselines, to the consequent counterpart properties of the baselines, to be preserved. Borrowing the term from Hokari and Thomson (2008), we refer to this aspect as assisted preservation.

Let us start with claims monotonicity. We say that an upper bound $b$ satisfies strong claims monotonicity if, for all $(N, c, E) \in \mathcal{D}$ and $i \in N, c_{i}<c_{i}^{\prime}$ implies $b_{i}\left(N,\left(c_{i}, c_{N \backslash\{i\}}\right), E\right)<$ $b_{i}\left(N,\left(c_{i}^{\prime}, c_{N \backslash\{i\}}\right), E\right)$ and $b_{j}\left(N,\left(c_{i}, c_{N \backslash\{i\}}\right), E\right)=b_{j}\left(N,\left(c_{i}^{\prime}, c_{N \backslash\{i\}}\right), E\right)$ for all $j \in N \backslash\{i\}$. It is not difficult to show that claims monotonicity of $R$ is assistedly preserved by the previous condition. More precisely, if $R$ is claims monotonic and $b$ is an upper bound that satisfies strong claims monotonicity, then $R^{b}$ satisfies claims monotonicity too. A straightforward consequence from 
here is that $O^{t}$ preserves claims monotonicity, as noted by Thomson and Yeh (2008).

We say that a lower bound $b$ satisfies strong linked monotonicity if, for all $(N, c, E) \in \mathcal{D}$ and $i \in N, b_{i}\left(N,\left(c_{i}+\varepsilon, c_{N \backslash\{i\}}\right), E+\varepsilon\right) \leq b_{i}(N, c, E)+\varepsilon$ and $b_{j}\left(N,\left(c_{i}+\varepsilon, c_{N \backslash\{i\}}\right), E+\varepsilon\right)=b_{j}(N, c, E)$ for all $j \in N \backslash\{i\}$. It is not difficult to show that linked monotonicity of $R$ is assistedly preserved by the previous condition. More precisely, if $R$ satisfies linked monotonicity and $b$ is an lower bound that satisfies strong linked monotonicity, then $R^{b}$ satisfies linked monotonicity too, a result that can be proved directly from the previous one on claims monotonicity by appealing to Theorem 5. A straightforward consequence from here is that $O^{m}$ preserves linked monotonicity, as noted by Thomson and Yeh (2008).

As for population monotonicity, we say that an upper bound satisfies extreme population monotonicity if, for all $(N, c, E) \in \mathcal{D}$ and $\left(N^{\prime}, c^{\prime}, E\right) \in \mathcal{D}$ such that $N \subseteq N^{\prime}$ and $c_{N}^{\prime}=c$, then $b_{i}\left(N^{\prime}, c^{\prime}, E\right)=b_{i}(N, c, E)$, for all $i \in N$. It is not difficult to see that if $R$ satisfies population monotonicity and $b$ is an upper bound that satisfies extreme population monotonicity, then $R^{b}$ satisfies population monotonicity too. Consequently, thanks to Theorem 5 , we also have that the dual of population monotonicity (i.e., resource-and-population monotonicity) is assistedly preserved by a lower bound $b$, provided this bound satisfies the dual property of extreme population monotonicity described above. Note that, as the truncated claims application satisfies extreme population monotonicity, it follows from the above that population monotonicity is preserved by the $O^{t}$, whereas resource-and-population monotonicity is preserved by the $O^{m}$, as noted by Thomson and Yeh (2008).

The previous discussion can be summarized as follows.

Proposition 3 Claims monotonicity, linked monotonicity, population monotonicity and resourceand-population monotonicity are assistedly preserved.

The following corollary, whose content is shown directly by Thomson and Yeh (2008) is a straightforward consequence of the above proposition and the preceding discussion.

Corollary 7 Claims monotonicity and population monotonicity are preserved by $O^{t}$, whereas linked monotonicity and resource-and-population monotonicity are preserved by $O^{m}$.

\subsection{No preservation}

The remaining properties described above are highly disruptive. This is certainly the case of the two composition properties, which might be somewhat surprisingly given the underlying 
connection between these properties and the composition operators. Something similar happens with resource monotonicity, consistency, resource-and-population uniformity, and self-duality. The reason for such a disruptive behavior in all these cases is that the effect of each of these properties on the baseline and the primitive rule cannot be disentangled, in contrast with the properties analyzed in the previous section.

We conclude referring to the properties dealing with the immunity of rules to coalitional manipulation. It is well known that the proportional rule is essentially the only non-manipulable rule (e.g., Ju et al., 2007). ${ }^{14}$ It follows from here that if the proportional rule is not a fixed point of a given composition operator, then such operator does not preserve any of the properties of non manipulability. That is certainly the case of $O^{m}$ and $O^{t}$, as mentioned by Thomson and Yeh (2008).

\section{References}

[1] Aumann RJ, Maschler M (1985) Game theoretic analysis of a bankruptcy problem from the Talmud, Journal of Economic Theory 36, 195-213.

[2] Dominguez, D. (2010) Lower bounds and recursive methods for the problem of adjudicating claims. ITAM Discussion Paper.

[3] Dominguez D., Thomson W., (2006) A new solution to the problem of adjudicating conflicting claims. Economic Theory 28, 283-307.

[4] Hokari, T., Thomson, W., (2008) On properties of division rules lifted by bilateral consistency. Journal of Mathematical Economics 44, 1057-1071.

[5] Hougaard, J.L., J.D Moreno-Ternero and L.P. Østerdal (2010), Baseline rationing, University of Copenhagen Discussion Paper 10-16.

[6] Ju, B.-G., Miyagawa E., Sakai T. (2007), Non-manipulable division rules in claim problems and generalizations, Journal of Economic Theory 132, 1-26.

[7] Moreno-Ternero J., Roemer J., (2006) Impartiality, solidarity, and priority in the theory of justice. Econometrica 74, 1419-1427.

\footnotetext{
${ }^{14}$ This is not only the case for manipulations via merging or splitting claims, but also with respect to any other similar form of manipulation such as reallocation or transfers of claims (e.g., Ju et al, 2007).
} 
[8] Moreno-Ternero J., Villar A., (2004) The Talmud rule and the securement of agents' awards. Mathematical Social Sciences 47, 245-257.

[9] Moreno-Ternero J., Villar A., (2006) The TAL-family of rules for bankruptcy problems. Social Choice and Welfare 27, 231-249.

[10] Moulin H., (2000) Priority rules and other asymmetric rationing methods. Econometrica $68,643-684$.

[11] Moulin H., (2002) Axiomatic Cost and Surplus-Sharing, Chapter 6 of K. Arrow, A. Sen and K. Suzumura (eds.), The Handbook of Social Choice and Welfare, Vol. 1. NorthHolland.

[12] O’Neill B., (1982) A problem of rights arbitration from the Talmud, Mathematical Social Sciences 2, 345-371.

[13] Thomson W., (1996) Consistent allocation rules, RCER Working paper 418, University of Rochester.

[14] Thomson W., (2003) Axiomatic and game-theoretic analysis of bankruptcy and taxation problems: a survey, Mathematical Social Sciences 45, 249-297.

[15] Thomson W., (2007), Fair allocation rules, RCER Working paper 539, University of Rochester.

[16] Thomson, W., C.-H. Yeh (2008), Operators for the adjudication of conflicting claims, Journal of Economic Theory 143, 177-198

[17] Young P (1988) Distributive justice in taxation, Journal of Economic Theory 44, 321335. 